Courrier du Centre International Blaise-

Pascal

38-39 | 2017-2018

Varia

\title{
Le siphon géant de Blaise Pascal
}

Armand Le Noxaïc et Pierre Lauginie

\section{CpenEdition}

Journals

Édition électronique

URL : https://journals.openedition.org/ccibp/1475

DOI : $10.4000 /$ ccibp. 1475

ISSN : 2493-7460

\section{Éditeur}

Centre international Blaise Pascal

\section{Édition imprimée}

Date de publication : 1 janvier 2019

Pagination : 161-174

ISBN : 978-2-84516-897-8

ISSN : 0249-6674

Référence électronique

Armand Le Noxaïc et Pierre Lauginie, «Le siphon géant de Blaise Pascal », Courrier du Centre International Blaise-Pascal [En ligne], 38-39 | 2017-2018, mis en ligne le 21 juin 2021, consulté le 03 mai 2022. URL : http://journals.openedition.org/ccibp/1475; DOI : https://doi.org/10.4000/ccibp.1475

Ce document a été généré automatiquement le 3 mai 2022.

Centre international Blaise Pascal 


\title{
Le siphon géant de Blaise Pascal
}

\author{
Armand Le Noxaïc et Pierre Lauginie
}

1 Le 8 octobre 1647, Blaise Pascal obtenait "permission" de faire imprimer Les expériences nouvelles touchant le vide. Cet opuscule était destiné à faire patienter ses lecteurs avant la parution d'un traité plus conséquent sur le vide. Pascal y précise que la publication de cet abrégé a pour but également de prendre de vitesse des auteurs qui pourraient publier sur ses expériences alors qu'il les a réalisées «avec beaucoup de frais, de peine et de temps ", et que le mérite lui en revient. Grâce à la première narration sur le vide de Roberval, nous savons que ces expériences auraient eu lieu en janvier et février 1647, dans la cour de la verrerie de Rouen. Dans son abrégé, Pascal y rapporte huit expériences dont certaines ont nécessité de très grands tubes longs de plus de $10 \mathrm{~m}$. Ces expériences à grande échelle étaient destinées à montrer qu'il était possible de créer des espaces vides, en utilisant n'importe quel fluide. En Italie, Torricelli venait de montrer qu'il était possible de le faire au-dessus d'une colonne de mercure. Pascal, après avoir refait l'expérience italienne avec succès, s'emploie à la faire avec de l'eau et d'autres fluides. La densité de l'eau 13.6 fois plus faible que celle $\mathrm{du}$ mercure, l'oblige à faire fabriquer et à manipuler des tubes de verre bien plus longs que ceux utilisés par le savant italien.

2 Parmi ces huit expériences, celle du siphon géant, la quatrième citée dans son opuscule, a dû être la plus spectaculaire. Pour la réaliser, Pascal aurait fait fabriquer par les excellents verriers de Rouen un siphon aux dimensions impressionnantes, avec des jambes de 50 et 45 pieds. Comme souvent, un doute plane sur la réalisation par Pascal de cette expérience ${ }^{1}$. Peu de temps après sa mort, Robert Boyle ${ }^{2}$, qui réalise de nombreuses expériences sur le vide, est sceptique sur la réalité de certaines expériences pascaliennes - elles seraient des expériences de pensée. Plus proche de nous, au début $\mathrm{du} \mathrm{xx}^{\mathrm{e}}$ siècle, Félix Mathieu ${ }^{3}$ publie plusieurs articles polémiques à ce sujet, mettant en doute la probité de Pascal. Puis, au milieu du $\mathrm{xx}^{\mathrm{e}}$ siècle, Alexandre Koyré accuse ${ }^{4}$ Pascal de ne pas avoir fait la troisième expérience qu'il cite dans son abrégé, nécessitant des tubes longs de 46 pieds. Plus proche de nous, dans les années 1980, la chercheuse japonaise Kimiyo Koyanagi ${ }^{5}$ affirme que Pascal n'a jamais 
fait la plupart des expériences qu'il cite dans ses traités de physique - dont évidemment les troisième et quatrième expériences nécessitant les plus longs tubes.

Cependant, il y a quelques années de cela, suite à une reconstitution de la troisième expérience pascalienne, nous avions pu retrouver les résultats expérimentaux ${ }^{6}$ consignés par Pascal et Roberval dans leurs écrits respectifs. Koyré et Koyanagi basaient leurs propos accusateurs sur des reconstitutions insuffisamment fidèles, selon notre avis, de cette expérience compliquée, qui montraient des résultats différents voire opposés à ceux de Pascal. Pour réussir notre reconstitution, nous dûmes interpréter des données historiques et scientifiques, trouvées dans la première narration sur le vide de Roberval, en paramètres expérimentaux - ce qui n'avait pas été fait auparavant. La question est de savoir s'il en serait de même lors d'une reconstitution de l'expérience 4 - celle du siphon géant. Nous commencerons par relater ce que dit Pascal de cette expérience en citant le court descriptif qu'il en donne, et en le commentant. Puis, nous exposerons les résultats d'une recherche historique sur les témoignages de l'époque autour de cette expérience. Ensuite, nous relaterons la reconstitution que nous en avons faite sur le site de la nouvelle Université Paris-Saclay. Pour finir nous exposerons les résultats obtenus, et conclurons.

\section{L'expérience commentée du siphon géant décrite par Blaise Pascal}

4 Comme à son habitude, Pascal fait de ses expériences un descriptif très épuré, en ne donnant que les détails qui lui semblent vraiment essentiels. Le texte relatif à l'expérience du siphon géant, que l'on trouve dans les Expériences nouvelles touchant le vide, n'échappe pas à cette règle et surtout à son style littéraire :

Un siphon scalène, dont la plus longue jambe est de cinquante pieds et la plus courte de quarante-cinq, étant rempli d'eau, et les deux ouvertures bouchées étant mises dans deux vaisseaux pleins d'eau et enfoncées environ d'un pied, en sorte que le siphon soit perpendiculaire à l'horizon et que la surface de l'eau d'un vaisseau soit plus haute que la surface de l'autre de cinq pieds: si l'on débouche les deux ouvertures, le siphon étant en cet état, la plus longue jambe n'attire point l'eau de la plus courte, ni par conséquent celle du vaisseau où elle est, contre le sentiment de tous les philosophes et artisans; mais l'eau descend de toutes les deux jambes dans les deux vaisseaux jusques à la même hauteur que dans le tuyau précédent, en comptant la hauteur depuis la surface de l'eau de chacun des vaisseaux. Mais ayant incliné le siphon au-dessous de la hauteur d'environ 31 pieds, la plus longue jambe attire l'eau qui est dans le vaisseau de la plus courte ; et quand on le rehausse audessus de cette hauteur, cela cesse, et tous les deux côtés dégorgent, chacun dans son vaisseau ; et quand on le rabaisse, l'eau de la plus longue jambe attire l'eau de la plus courte comme auparavant ${ }^{7}$.

5 Les paramètres expérimentaux tirés de ce court descriptif sont toutefois assez nombreux. La plus longue jambe du siphon mesure environ $16 \mathrm{~m} \mathrm{20}$, la plus courte environ $14 \mathrm{~m}$ 60. Le fluide utilisé est de l'eau. Le siphon est initialement rempli d'eau et bouché à chacune de ses deux extrémités. Il se trouve au début de l'expérience dans une position verticale par rapport au sol. Chacune de ses extrémités est plongée d'environ $30 \mathrm{~cm}$ dans un récipient contenant de l'eau. Cependant, du fait de la différence de longueur des deux jambes du siphon, la surface de l'eau d'un des récipients se situe environ $1 \mathrm{~m} 60$ au-dessus de celle du récipient inférieur. Les deux extrémités sont débouchées simultanément, le siphon se désamorce instantanément. 
L'eau descend dans chacune des jambes pour se stabiliser à une hauteur de $10 \mathrm{~m}$ environ par rapport à la hauteur de la surface de l'eau dans chaque récipient.

Ensuite, Pascal incline la structure du siphon, pour que son sommet descende sous $10 \mathrm{~m}$ de hauteur, par rapport à quoi ? Pascal ne le dit pas, et son texte semble nous donner des indices contradictoires quant à la position du sommet du siphon. Lorsque le sommet du siphon est incliné en-dessous de $10 \mathrm{~m}$, il dit que «l'eau de la plus longue jambe attire l'eau de la plus courte ». Cela laisse penser que la jambe la plus longue est remplie d'eau, comme l'est celle plus courte. Or, si l'on reste dans la logique de son propos, cela veut dire que la hauteur du sommet du siphon est inférieure à $10 \mathrm{~m}$ par rapport aux niveaux d'eau des deux récipients. Par conséquent, cela signifierait que Pascal situerait la hauteur critique de $10 \mathrm{~m}$ par rapport à la surface de l'eau du réservoir inférieur, ou par rapport au sol. D'ailleurs, il semble sous-entendu au prime abord qu'il situe le sommet du siphon par rapport au niveau du sol, puisqu'il n'apporte pas davantage de précisions. Oui, mais pourquoi alors dit-il que si on élève le sommet du siphon au-dessus des $10 \mathrm{~m}$, les deux côtés dégorgent? Si les $10 \mathrm{~m}$ étaient considérés par rapport au réservoir inférieur, seul le tube le plus long dégorgerait, et non pas les deux. Et ceci, tant que les hauteurs d'eau n'auraient pas dépassé la valeur critique de $10 \mathrm{~m}$ dans les deux jambes. Cette hauteur critique de $10 \mathrm{~m}$ ne serait-elle pas considérée par rapport au réservoir supérieur?

7 Son descriptif de l'expérience 7 dans son opuscule peut nous apporter des éclaircissements. En effet cette expérience est similaire à l'expérience 4 du siphon géant, mais elle utilise du vif-argent (du mercure) au lieu de l'eau. De ce fait, elle nécessite un siphon de plus petites dimensions. Pascal dit :

Ayant rempli un siphon de vif-argent, dont la plus longue jambe a 10 pieds, et l'autre neuf et demi, et mis les deux ouvertures dans deux vaisseaux de vif-argent, enfoncées environ d'un pouce chacune, en sorte que la surface du vif-argent de l'un soit plus haute d'un demi-pied que la surface de vif-argent de l'autre; quand le siphon est perpendiculaire, la plus longue jambe n'attire pas le vif-argent de la plus courte; mais le vif-argent, se rompant par le haut, descend dans chacune des jambes, et regorge dans les vaisseaux, et tombe jusques à la hauteur ordinaire de deux pieds trois pouces, depuis la surface du vif-argent de chaque vaisseau. Que si on incline le siphon, le vif-argent des vaisseaux remonte dans les jambes, les remplit, et commence de couler de la jambe la plus courte dans la plus longue, et ainsi vide son vaisseau; car cette inclination dans les tuyaux où est ce vide apparent, lorsqu'ils sont dans quelque liqueur, attire toujours les liqueurs des vaisseaux, si les ouvertures des tuyaux ne sont point bouchées, ou attire le doigt, s'il bouche ces ouvertures ${ }^{8}$.

8 L'expérience est similaire à celle de l'eau dans le déroulement, mais Pascal y apporte une information supplémentaire. Il précise que lorsque l'on incline le siphon, le liquide remonte dans les jambes, puis les remplit, avant de couler de la plus courte jambe vers la plus longue. Il ne précisait pas pour l'expérience 4, que les jambes se remplissaient toutes les deux, avant que le siphon fonctionne de nouveau. Or, dans la logique de sa position par rapport à l'existence du vide, la plus longue jambe ne pourra se remplir seulement si on incline le sommet du siphon d'une hauteur inférieure à la hauteur critique de 2 pieds trois pouces de mercure. Cette précision de Pascal nous apporte deux informations importantes pour notre propos. La première est que la hauteur critique de liquide, 31 pieds pour l'eau, ou 2 pieds 3 pouces pour le mercure, est bien considérée par rapport au récipient inférieur. La seconde information est, qu'à l'époque des expériences de Rouen en 1647 et de la rédaction de cet opuscule la même année, 
Pascal n'a pas encore fait le lien entre les effets de la pression atmosphérique et des hauteurs critiques de ces liquides. Car, sinon il aurait envisagé que l'air extérieur exerçant une pression à la surface des liquides se trouvant dans les réservoirs aurait permis un transfert de liquide de la plus courte jambe vers la plus longue, même si cette dernière n'était pas remplie.

\section{Les témoignages et commentaires des contemporains de cette expérience}

9 Si cette expérience du siphon géant a bien été faite à Rouen, en début d'année 1647, on peut envisager qu'il y eut de nombreux spectateurs. On pense évidemment au professeur de philosophie Jacques Pierius et au médecin Pierre Guiffart, des notables rouennais, auteurs tous les deux d'un ouvrage sur le vide. Jacques Pierius publie An detur vacuum in rerum natura, à l'automne 1646. Peut-être présent lors de l'expérience du siphon géant de Pascal, il n'a pu donc s'y référer dans son ouvrage qui lui est antérieur. Pierius adopte une position aristotélicienne, et le rejet de l'existence du vide par le philosophe grec. Néanmoins, il est intéressant de noter qu'il évoque, dans sa dissertation sur le vide, l'idée d'un siphon géant ${ }^{9}$. Pierre Guiffart commente cette idée de siphon géant de Pierius, dans son Discours $d u v^{v i d e}{ }^{10}$, achevé d'imprimer le 19 août 1647 :

Il nous reste encore à examiner quelque expérience que Mr Pierius met en avant, quand il dit: que si on prend deux canaux chacun de cent pieds de hauteur, dont l'un toutefois soit plus long quelque peu que l'autre, et qu'on les ajuste de sorte qu'étant unis ensemble ils fassent une forme d'angle, qu'on bouche leurs extrémités, et que par un trou fait en haut, qui serait commun à l'un et à l'autre, on $\mathrm{y}$ verse de l'eau, jusqu'à ce qu'ils soient pleins, puis qu'on bouche le trou d'en haut, et qu'on mette le bout le plus court dans une eau dormante, et qu'ensuite on les débouche tous deux : toute cette eau dormante, dit-il, montera de sorte qu'elle se videra par le côté le plus long ${ }^{11}$.

Plus loin dans son discours sur le vide, Guiffart fait allusion - à plusieurs reprises - à un siphon qui aurait moins de 33 pieds de hauteur :

Comme nous avons appris par les expériences de M. Pascal, si on prend une sarbatane de verre de plus de trente-trois pieds de longueur, bouchée très exactement par un bout, ou sigillée du sceau de Hermès, qu'on l'emplisse entièrement d'eau, puis qu'on en bouche très justement le bout avec le doigt, et qu'après l'avoir élevée perpendiculairement dans un vaisseau plein d'eau, de sorte que le bout sigillé soit en haut, et celui qu'on tient bouché soit dans l'eau, si après cela on ôte le doigt, l'eau ayant son passage libre descend de son canal jusques à la hauteur de trente-trois pieds, le surplus du canal paraissant vide ${ }^{12}$.

Or Pierius n'évoquait pas cette hauteur critique, et pour cause son ouvrage est publié avant les expériences de Pascal. Par conséquent, Guiffart ne peut insister sur cette hauteur sans s'inspirer de ce qu'a fait ou dit Blaise Pascal. Remarquons que le texte de Guiffart est antérieur de quelques mois à celui des Expériences nouvelles touchant le vide, publié par Pascal. Il est envisageable que Pascal se soit référé à Guiffart, dans le texte introductif de ses Expériences nouvelles : «j'ai craint qu'un autre qui n'y aurait employé le temps, l'argent, ni la peine, me prévenant, donnât au public des choses qu'il n'aurait pas vues, et lesquelles par conséquent il ne pourrait pas rapporter avec l'exactitude et l'ordre nécessaire pour les déduire comme il faut: n'y ayant personne qui ait eu des tuyaux et des siphons de la longueur des miens, et peu qui voulussent se donner la 
peine nécessaire pour en avoir $^{13} »$. Guiffart dit cependant avoir été invité par Pascal à certaines de ses expériences: "Monsieur Pascal, ayant fait depuis quelques temps plusieurs expériences en cette ville en la présence de tous les plus savants hommes de sa connaissance, me fit aussi la faveur de me convier aux deux dernières ${ }^{14}$ ». Mais, il ne précise pas quelles sont ces expériences - la numérotation des expériences indiquée par Pascal dans son opuscule sur le vide n'est pas obligatoirement celle de leur déroulement. Quoi qu'il en soit, il se réfère explicitement à cette expérience, lorsqu'il déclare: "Monsieur Pierius veut que l'eau dans laquelle trempe le côté de cent pieds monte par celui-ci, pour descendre par l'autre de cent et un pieds. Toutefois en un bien moindre siphon, l'expérience fait voir que cela n'arrive point, mais que par l'un et l'autre côté du siphon, l'eau descend jusqu'à la hauteur de trente-trois pieds ${ }^{15}$ ». Guiffart se réfère à une expérience bien réelle, qui ne peut être que celle de Pascal, dont il a été spectateur ou dont il en a été informé à Rouen.

11 Le témoignage de Guiffart a le plus de poids, car les autres commentaires qu'en fait Gassendi dans ses Animadversiones in decimum Librum Diogenis Laërtii, ou Roberval dans sa Narration à Desnoyers sur le vide, sont des témoignages indirects. Gassendi et Roberval n'étaient pas à Rouen, lors des expériences sur le vide faites par Pascal. Gassendi déclare en se référant à Pascal :

Ainsi en ayant fait confectionner divers de ce genre, et même un dont une jambe avait cinquante pieds et l'autre quarante-cinq, il découvrit qu'il ne se faisait aucun écoulement du vif-argent ni de l'eau qui le remplissait de la jambe la plus courte dans la plus longue, et du vase où plongeait l'orifice de celui-là jusqu'au vase où plongeait l'origine de celui-ci, à moins que la perpendiculaire menée du sommet, situé soit à la verticale, soit en oblique, jusqu'au plan du vif-argent ou de l'eau étendus en nappe dans le vase correspondant, ne dépassât, dans le cas du vifargent, à peu près deux pieds, trois pouces et demi, et, dans le cas de l'eau, environ trente-deux pieds ${ }^{16}$.

Quant à la narration de Roberval, il s'agit d'une lettre datée du 20 septembre 1647, antérieure de peu à la permission d'imprimer de Pascal pour son opuscule sur le vide. Après avoir évoqué le siphon réalisé par Pascal pour le mercure, il se réfère à celui réalisé pour de l'eau: "Que l'on n'aille donc pas penser à l'avenir que d'un versant d'une montagne aussi haute qu'on voudra, l'eau puisse, grâce à un siphon très puissant, franchir le sommet de la montagne et redescendre dans la vallée située au versant opposé de la montagne: car cela ne peut se faire au-dessus d'une hauteur de 31 pieds ${ }^{17} »$.

Ces écrits semblent attester de la réalité de cette expérience du siphon géant. Insistons cependant sur l'absence de leurs auteurs lors de cette expérience du siphon géant, sauf peut-être Pierre Guiffart, mais sans certitude puisqu'il n'était présent qu'à deux expériences de Pascal. Si Pascal a respecté le déroulement de ses expériences dans la numérotation de celles-ci dans son opuscule, Guiffart aurait assisté aux $7^{\mathrm{e}}$ et $8^{\mathrm{e}}$ expériences, qui étaient déjà très spectaculaires. Pour celles-ci, Pascal emploie un siphon de plus petites dimensions - 10 pieds pour la plus longue jambe et 9.5 pieds pour la plus courte. Cette hypothèse est très envisageable, Guiffart aurait appris ce jour-là l'expérience faite précédemment avec un siphon géant et de l'eau. 


\section{Les reconstitutions de l'expérience du siphon géant}

13 On pouvait se demander si, en refaisant l'expérience du siphon géant, nous allions retrouver les résultats expérimentaux de Blaise Pascal, et pouvoir conforter, ou au contraire fragiliser, l'idée que cette expérience pascalienne était davantage qu'une expérience de pensée.

Cependant, une reconstitution ne peut apporter des éléments fiables, seulement si elle est suffisamment fidèle à l'expérience d'origine. C'est une pratique difficile si on veut avoir ce niveau d'exigence. C'est pourquoi, entre 2012 et 2017, furent entreprises plusieurs reconstitutions du siphon géant, sur le site de la nouvelle université ParisSaclay.

15 La première reconstitution ${ }^{18}$ montra un phénomène qui semblait contredire le compte rendu de Pascal de cette expérience. Si l'on amorçait le siphon lorsque son sommet se situait à environ $7 \mathrm{~m} 40 \mathrm{du}$ sol, et que l'on élevait son sommet progressivement, il ne se désamorçait pas même si l'on dépassait la hauteur critique indiquée par Pascal d'environ $10 \mathrm{~m}$. L'eau continuait de s'écouler du récipient le plus haut vers celui le plus bas. Le dispositif permettait de hisser ce sommet à $11 \mathrm{~m} 50$ par rapport au sol, mais pas davantage; le siphon fonctionna toujours même à cette hauteur. A préciser que la différence des hauteurs entre les deux cuves était de $3 \mathrm{~m} 40$, alors que dans l'expérience décrite par Pascal elle était de $1 \mathrm{~m} 60$. Ce paramètre divergent ne pouvait-il pas expliquer ce résultat contradictoire?

Fig. 1.

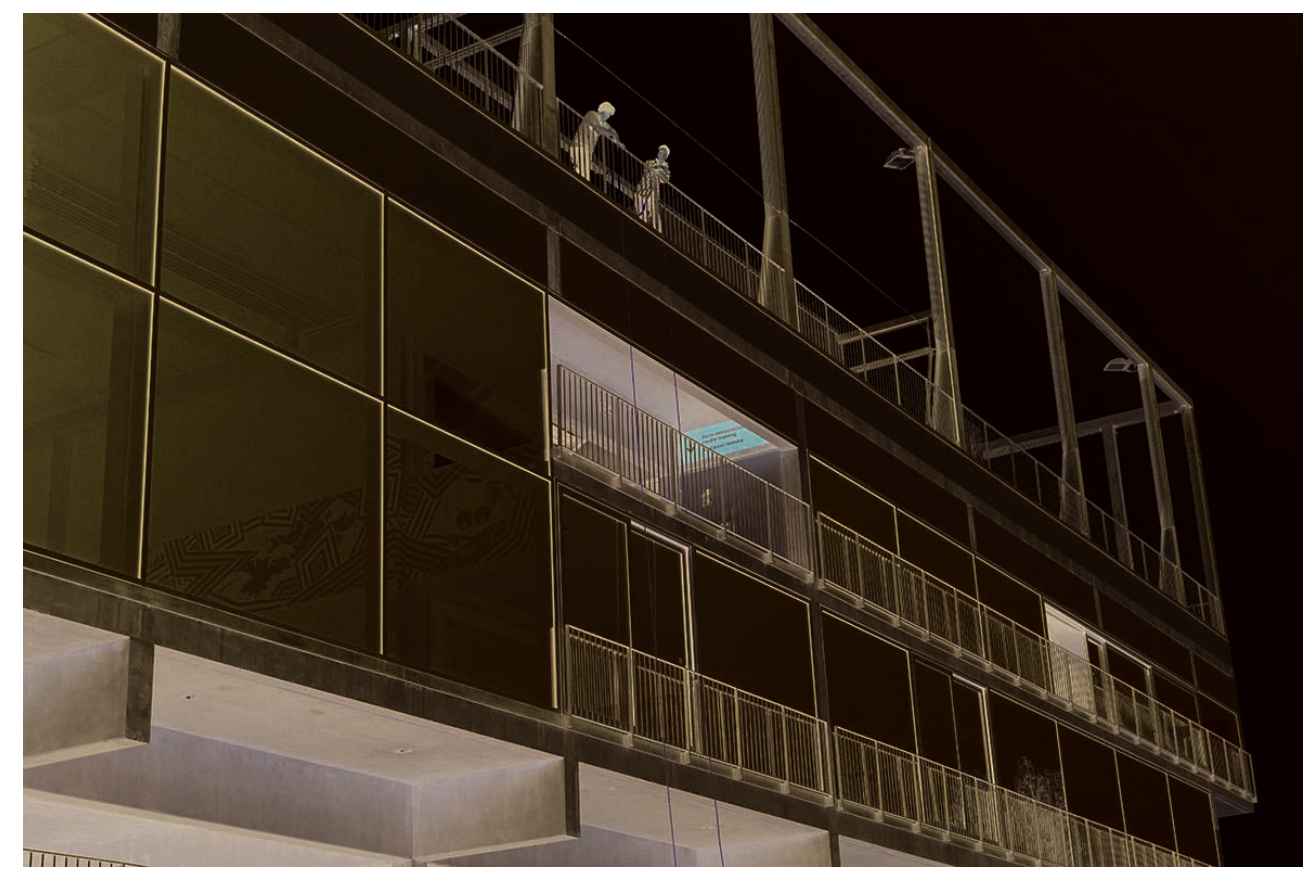

Lors de la seconde reconstitution ${ }^{19}$, le sommet du siphon, rempli d'eau et bouché à ses deux extrémités, se situait à $11 \mathrm{~m} 50$ par rapport à la cuve la plus basse, et $10 \mathrm{~m}$ par rapport à celle la plus haute. Cette fois la différence de hauteur entre les cuves était très proche de celle indiquée par Pascal. Les extrémités furent débouchées, et le siphon ne put s'amorcer et de l'eau descendit de chacune des jambes, à des hauteurs qu'il était difficile d'évaluer, en raison des conditions de sécurité de l'expérience. Ce résultat 
semblait néanmoins confirmer les dires de Pascal. Si le sommet du siphon dépassait une hauteur critique d'environ $10 \mathrm{~m}$, le siphon ne peut s'amorcer. Alors, pourquoi étionsnous parvenus à faire fonctionner le siphon à $11 \mathrm{~m} 50$, la première fois ? D'autres essais complémentaires semblaient nécessaires.

17 Pour la troisième reconstitution ${ }^{20}$, le but était de savoir jusqu'à quelle hauteur le siphon restait amorcé lorsque l'on hissait son sommet durant l'écoulement. La différence de hauteur entre les cuves était de $1 \mathrm{~m}$. Le siphon fut amorcé lorsque son sommet était à

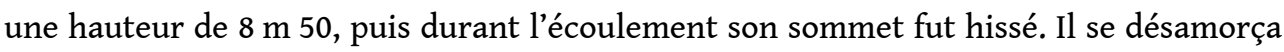
complètement lorsque son sommet se situa à environ $14 \mathrm{~m}$ 90, après que l'écoulement ait eu des ratés pour une hauteur d'environ $12 \mathrm{~m}$ 90. Ce résultat confirma celui de 2012 ; lorsque le sommet du siphon est hissé, pendant qu'il est amorcé, il est possible de dépasser de quelques mètres la hauteur indiquée par Pascal.

La quatrième reconstitution ${ }^{21}$ avait pour but de vérifier le résultat de la seconde reconstitution. La différence de hauteur entre les cuves était de $1 \mathrm{~m} \mathrm{50.} \mathrm{Le} \mathrm{sommet} \mathrm{du}$ siphon se situait à $10 \mathrm{~m} 66$ par rapport au sol, et à $9 \mathrm{~m} 16$ par rapport à l'eau contenue dans la cuve la plus haute. Les extrémités du siphon rempli d'eau furent débouchées, le siphon fonctionna durant presque 4 minutes, puis se désamorça. En étant un peu audessus de la hauteur critique indiquée par Pascal, le siphon pouvait fonctionner durant un temps limité. Le sommet du siphon fut abaissé et réamorcé, à $9 \mathrm{~m}$ de hauteur par rapport au sol. Cette opération avait été pratiquée avec succès lors de toutes les reconstitutions - ce qui confirmait les dires de Pascal à ce sujet ${ }^{22}$.

Il restait à savoir ce qu'il se passerait précisément si le sommet du siphon se situait à une hauteur proche de celle indiquée par Pascal, soit environ $16 \mathrm{~m}$. Ce fut l'objet de la cinquième reconstitution ${ }^{23}$ Les étages intermédiaires du bâtiment nous permettraient de mieux observer et de faire des mesures précises des hauteurs de liquide dans le siphon s'il se désamorçait, que lors de la seconde reconstitution. Le sommet du siphon se situait $15 \mathrm{~m} 22$ au-dessus du niveau d'eau de la cuve se trouvant au sol, et $13 \mathrm{~m} 82$ par rapport à celui de la cuve la plus haute. La différence de hauteur entre les deux cuves était de $1 \mathrm{~m}$ 53. La configuration du siphon était très proche de celle indiquée par Pascal dans son descriptif. Les extrémités plongeant dans l'eau contenue par les cuves furent débouchées simultanément. Le siphon ne put s'amorcer. Chacune des deux jambes du siphon dégorgea dans les cuves, et une colonne d'eau resta en suspension dans chacune des jambes. Dans la plus longue jambe, fut mesurée une colonne d'eau de $9 \mathrm{~m} 90$, dans la plus petite une colonne de $9 \mathrm{~m} \mathrm{72}$. Soient des valeurs très proches de la hauteur critique d'environ $10 \mathrm{~m}$ indiquée par Pascal.

\section{Analyse de ces reconstitutions}

20 À partir de l'ensemble de ces reconstitutions, nous avons des éléments nouveaux sur les différentes étapes de l'expérience décrite par Pascal. Reconsidérons-la selon le déroulement indiqué par Pascal. Initialement, le siphon géant se trouve dans la configuration de la $5^{\mathrm{e}}$ reconstitution; et nous avons constaté la même chose que lui. Le siphon se désamorce immédiatement, les jambes dégorgent, et les niveaux d'eau se stabilisent à des hauteurs proches de $10 \mathrm{~m}$ par rapport aux niveaux d'eau dans les cuves. Puis Pascal dit incliner le siphon, pour que son sommet se situe à moins de $10 \mathrm{~m}$, et que le siphon se réamorce. Nous l'avons en effet constaté à chacune des reconstitutions. Il rehausse ensuite le siphon, qui se désamorce de nouveau lorsque son 
sommet dépasse la hauteur critique d'environ $10 \mathrm{~m}$. Nous avons constaté que cela se produisait à une hauteur supérieure de quelques mètres. Mais vers $13 \mathrm{~m}$ de hauteur l'écoulement a des ratés, et ce serait peut-être arrêté si nous avions laissé plus longtemps le siphon dans cette même configuration. Nous ignorons à quelle vitesse Pascal a hissé le siphon. Il est envisageable qu'assez rapidement, le dispositif ait été remis dans la configuration initiale, et que le siphon se soit désamorcé en très peu de temps. Allons même plus loin dans cette idée. Nous avons constaté que le siphon continuait de fonctionner lorsque nous attenions les $13 \mathrm{~m}$ avec des ratés, pour se désamorcer vers $14 \mathrm{~m}$ 90. Pascal a probablement observé la même chose que nous lors de ses expériences. Aussi, peut-être n'est-ce pas un hasard si Pascal emploie des tubes longs de plus de $15 \mathrm{~m}$ - une longueur, et donc une hauteur, pour laquelle il sait que le siphon se désamorcera complètement.

Cependant, nous avons pu constater, en accord avec ce que décrit Pascal, qu'il y a bien une hauteur critique d'eau, qui lorsqu'elle est dépassée conduit au désamorçage du siphon, plus ou moins rapide. Le nombre important de ces reconstitutions ont apporté des éléments supplémentaires. Lors de la $4^{\mathrm{e}}$ reconstitution, nous avons débouché le siphon lorsque son sommet se situait un peu au-dessus de la hauteur critique des $10 \mathrm{~m}$, par rapport à la cuve inférieure, mais autour de $9 \mathrm{~m}$ par rapport à la cuve supérieure. Le siphon a fonctionné pendant quasiment 4 minutes, puis s'est désamorcé. La hauteur indiquée par Pascal est en effet une hauteur critique, pour laquelle le siphon peut fonctionner seulement pendant un temps limité, ou avoir des ratés avant que l'écoulement cesse.

\section{Au regard des connaissances actuelles}

La physique contemporaine explique les nuances constatées avec le récit expérimental de Pascal. Le phénomène de cohésion moléculaire explique pourquoi le siphon continue de fonctionner lorsque l'on dépasse quelque peu la hauteur critique. Les forces attractives de Van der Waals entre les molécules d'eau font qu'elles s'entraînent un peu comme les maillons d'une chaîne. Cependant ce phénomène de cohésion moléculaire ne permet pas de faire fonctionner le siphon pour une hauteur dépassant de beaucoup la hauteur critique. Car celle-ci résulte du calcul de la hauteur de la colonne d'eau dont la pression compenserait la pression atmosphérique extérieure. Si l'on dépasse trop cette hauteur critique, la "chaîne " des molécules d'eau se brise, et les jambes du siphon dégorgent. De plus, la cohésion moléculaire permet que l'écoulement se fasse au-dessus de la hauteur critique, d'autant plus que le fluide est déjà en mouvement. À cette condition, il est possible de dépasser la hauteur critique de quelques mètres. Dans le cas contraire, si le fluide est au repos, il ne peut se mettre en mouvement, au-dessus de la hauteur critique indiquée par Pascal de seulement quelques dizaines de centimètres. Par ailleurs, nous savons aujourd'hui qu'il y a une pression de vapeur saturante audessus des colonnes de l'eau qui reste dans chaque jambe du siphon une fois qu'il s'est désamorcé. Cette pression de vapeur d'eau correspondant à environ $24 \mathrm{~cm}$ d'eau liquide. C'est pourquoi, nous avons mesuré des hauteurs d'eau un peu inférieures à celle de $10 \mathrm{~m}$ rapportée par Pascal. 


\section{Conclusion}

Que ce soit par l'analyse des textes historiques ou grâce à ces différentes reconstitutions, tout semble indiquer que Blaise Pascal a bien fait cette expérience du siphon géant. En effet, Pierre Guiffart évoque, dans son ouvrage antérieur à celui de Pascal, un siphon de 33 pieds, en se référant aux expériences de l'illustre savant et en vantant ses mérites. Il se réfère également à d'autres expériences que consignera Pascal dans son opuscule sur le vide. On ne peut envisager Guiffart imaginer les détails de toutes ces expériences, et vouloir - qui plus est - les attribuer à un autre. Les reconstitutions ont montré des phénomènes analogues à ceux décrits par Pascal, avec des nuances qui peuvent se justifier. Cela conforte notre position qui est de croire que la probité de Blaise Pascal expérimentateur a été injustement mise à mal, notamment par Alexandre Koyré. Les reconstitutions des deux expériences les plus spectaculaires de Rouen ont toutes deux montré des résultats très proches de ceux consignées par Pascal ${ }^{24}$.

Par ailleurs, cette étude appuie l'idée que Pascal n'avait pas encore fait le lien entre le vide et la pression atmosphérique, à l'époque des expériences de Rouen. Cela viendra peu de temps après - ce qui le conduira à commanditer l'expérience du Puy-de-Dôme. Concernant celles de Rouen, Pascal a simplement calculé la hauteur critique d'eau seulement à partir de la hauteur de la colonne de mercure dans l'expérience de Torricelli qu'il avait refait avec succès, et le rapport de densité entre le mercure et l'eau.

\section{BIBLIOGRAPHIE}

BOYLE, Robert, The works of Robert Boyle, t. 5, edited by Michael Hunter and Edward B. Davis, Pickering and Chatto, London, 1999.

GUIFFART, Pierre, Discours du vuide, sur les expériences de M. Paschal et le traité de M. Pierius, J. Besongne, Rouen, 1648.

KOYANAGI, Kimiyo, «Cet effrayant petit livret... Expériences nouvelles touchant le vide de Blaise Pascal », dans Les Pascal à Rouen 1640-1648, Publications de l'Université de Rouen, 2001, p. 137-157.

KOYRÉ, Alexandre, Pascal, l'homme et l'œuvre, Cahiers de Royaumont, Philosophie $n^{\circ} 1$, Éd. de Minuit, 1956, p. 273-278.

LE NOXAÏC, Armand, «Comment Blaise Pascal a pu envisager et réaliser l'expérience des liqueurs de Rouen », Revue d'Histoire des Sciences, t. 68, 2015/1.

LE NOXAÏC, Armand, « Le crève-tonneau de Pascal : mythe ou réalité ? ", dans À quoi servent les instruments scientifiques?, Pulim, 2017.

LE NOXAÏC, Armand et LAUGINIE, Pierre, « Reconstitution de l'expérience des liqueurs de Blaise Pascal », Courrier du Centre International Blaise Pascal, $\mathrm{n}^{\circ}$ 32, 2010.

MATHIEU, Félix, Revue de Paris, $1^{\mathrm{er}}$ avril 1906, 15 avril 1906, $1^{\mathrm{er}}$ mars 1907. 
MESNARD, Jean, CEuvres complètes de Pascal, t. 2, Desclée de Brouwer (quatre tomes parus, 1964-1992).

\section{NOTES}

1. À l'inverse - paradoxalement - l'expérience du crève-tonneau est attribuée à Blaise Pascal, alors qu'il ne la revendique pas. Une étude récente a montré que l'expérience du crève-tonneau était un mythe à plusieurs niveaux. Armand Le Noxaïc, «Le crèvetonneau de Pascal : mythe ou réalité ? ", dans A quoi servent les instruments scientifiques?, Limoges, Pulim, 2017.

2. Robert Boyle, The works of Robert Boyle, t. 5, edited by Michael Hunter and Edward

B. Davis, London, Pickering and Chatto, 1999, p. 206.

3. Félix Mathieu, Revue de Paris, $1^{\mathrm{er}}$ avril 1906, 15 avril 1906, $1^{\mathrm{er}}$ mars 1907.

4. Alexandre Koyré, Pascal, l'homme et l'œuvre, Cahiers de Royaumont, Philosophie $\mathrm{n}^{\circ} 1$, Paris, Éd. de Minuit, 1956, p. 273-278.

5. Kimiyo Koyanagi, «Cet effrayant petit livret... Expériences nouvelles touchant le vide de Blaise Pascal », dans Les Pascal à Rouen 1640-1648, Rouen, Publications de l'Université de Rouen, 2001, p. 137-157.

6. Armand Le Noxaïc, Pierre Lauginie, « Reconstitution de l'expérience des liqueurs de Blaise Pascal», Courrier du Centre International Blaise Pascal, $\mathrm{n}^{\circ} 32,2010$; Armand Le Noxaïc, «Comment Blaise Pascal a pu envisager et réaliser l'expérience des liqueurs de Rouen ", Revue d'Histoire des Sciences, t. 68, 2015/1.

7. Jean Mesnard, Euvres complètes de Pascal, t. 2, Paris, Desclée de Brouwer (quatre tomes parus, 1964-1992), p. 503.

8. Ibid., t. 2, p. 504-505.

9. Jacques Pierius, An detur vacuum in rerum natura, BnF R-13514.

10. Pierre Guiffart, Discours du vuide, sur les expériences de M. Paschal et le traité de M. Pierius, J. Besongne, Rouen, 1648. BnF R-13513.

11. Ibid., p. 176-177.

12. Ibid., p. 37-38.

13. Jean Mesnard, op. cit., t. 2, p. 501.

14. Pierre Guiffart, op. cit., p. 7.

15. Ibid., p. 180-181.

16. Jean Mesnard, op. cit., t. 2, p. 725.

17. Ibid., p. 475.

18. Expérience faite en 2012 sur le site de l'IUT d'Orsay, avec la collaboration des étudiants Jérémy Gomes et Salah Ismail. La Pression atmosphérique était de $957 \mathrm{hPa}$. Le tuyau utilisé était en PVC souple, de diamètre intérieur $12 \mathrm{~mm}$.

19. Expérience faite au printemps 2016, du haut du toit de l'école Polytech Paris-Sud, avec la collaboration des étudiants Caroline Jedrzejczyk, Hugo Potier, et Marin Prieur. Le tuyau utilisé était en PVC souple, de diamètre intérieur $6 \mathrm{~mm}$. Le même tuyau fut utilisé, par la suite, pour les autres reconstitutions. 
20. Réalisée durant l'été 2016 , du haut de la terrasse du Laboratoire de Physique des Solides de l'UPsud, avec la collaboration de Pierre Lauginie, et les étudiants Caroline Jedrzejczyk, Hugo Potier, et Marin Prieur. Patm = $957 \mathrm{hPa}$.

21. Réalisée à l'automne 2016, du haut de l'avant-dernier étage du nouveau «lieu de vie » de l'UPSaclay, avec la collaboration de Pierre Lauginie, et les étudiants Nadir Azizi, Vincent Malgorn, et Thomas Michard. Patm $=1017 \mathrm{hPa}$.

22. Un reportage de cette expérience fut diffusé sur France Culture, lors de l'émission de la Méthode scientifique, intitulée Blaise Pascal: le pari scientifique, le $1^{\text {er }}$ décembre 2016.

23. Réalisée en mars 2017, du haut du dernier étage du «lieu de vie» de l'UPSaclay, avec la collaboration de Laurent Nagat, et les étudiants Jun Sun, Haojun Zhu, et Pin Lyu. Patm $=1003 \mathrm{hPa}$.

24. Celle du siphon géant exposée ici, et celle de l'expérience des liqueurs. Armand Le Noxaïc, Pierre Lauginie, "Reconstitution de l'expérience des liqueurs de Blaise Pascal », art. cit. ; Armand Le Noxaïc, « Comment Blaise Pascal a pu envisager et réaliser l'expérience des liqueurs de Rouen », art. cit.

INDEX

Mots-clés : vide, expérience, siphon géant

Keywords : vacuum, experience, giant siphon

\section{AUTEURS}

\section{ARMAND LE NOXAÏC}

Laboratoire Étude sur les sciences et les techniques (EST), Université Paris-Sud Orsay

PIERRE LAUGINIE

Laboratoire Étude sur les sciences et les techniques (EST), Université Paris-Sud Orsay 